\title{
A Method of Prolongation of Tangential Cauchy-Riemann Equations
}

\author{
Chong-Kyu Han and Jae-Nyun Yoo*
}

\section{$\S 0$. Introduction}

In this paper we present a method of prolongation of tangential Cauchy-Riemann equations. The technique is, roughly speaking, separating the holomorphic derivatives of CR functions from their complex conjugates and applying the tangential Cauchy-Riemann operators to the holomorphic part. Using this method we show that under generic assumptions mappings of a CR manifold into a CR manifold of higher dimension satisfy a certain Pfaffian system in the jet space, which implies the rigidity and the regularity of CR mappings.

Let $M$ be a differentiable manifold of dimension $2 m+1$. A CR structure on $M$ is a subbundle $\mathcal{V}$ of the complexified tangent bundle $T_{\mathbb{C}} M$ having the following properties:

i) each fiber is of complex dimension $m$,

ii) $\mathcal{V} \cap \overline{\mathcal{V}}=\{0\}$,

iii) $[\mathcal{V}, \mathcal{V}] \subset \mathcal{V}$ (integrability).

It is well known that if $(M, \mathcal{V})$ is real analytic $\left(C^{\omega}\right) M$ is locally embeddable into $\mathbb{C}^{m+1}$ as a real hypersurface. In this paper we are concerned with $\mathrm{CR}$ mappings of $M$ into a $C^{\omega}$ real hypersurface $N$ of $\mathbb{C}^{n+1}, n \geq m$. Let $N$ be a $C^{\omega}$ real hypersurface of nondegenerate Levi form in $\mathbb{C}^{n+1}$ defined by $r(z, \bar{z})=0$, where $z=\left(z_{1}, \cdots, z_{n+1}\right)$. Let $A$ and $B$ be $(n+1)$-tuple of nonnegative integers and let $z^{A}=z_{1}^{a_{1}} \cdots z_{n+1}^{a_{n+1}}$ if $A=\left(a_{1}, \cdots, a_{n+1}\right)$. After a holomorphic change of coordinates $r(z, \bar{z})$ can be written as

$$
r(z, \bar{z})=z_{n+1}+\bar{z}_{n+1}+\sum_{j=1}^{n} \lambda_{j} z_{j} \bar{z}_{j}+\sum_{A, B} c_{A B} z^{A} \bar{z}^{B}
$$

Received September 21, 1995

Revised December 11, 1995

*Both authors were supported by GARC-KOSEF 1995 
where each $\lambda_{j}$ is either 1 or -1 and each term in the last summand is of weight $\geq 3$. Weight of a term $c_{A B} z^{A} \bar{z}^{B}$ is $\sum_{j=1}^{n}\left(a_{j}+b_{j}\right)+2\left(a_{n+1}+b_{n+1}\right)$ as in [Ch-M]. Now let $\left\{L_{1}, \cdots, L_{m}\right\}$ be a local basis for $\mathcal{V}$. A mapping $f=\left(f_{1}, \cdots, f_{n+1}\right): M \rightarrow N$ is a CR mapping if and only if

$$
\bar{L}_{i} f_{j}=0, \quad i=1, \cdots, m, \quad j=1, \cdots, n+1
$$

(tangential Cauchy-Riemann equations).

For an $m$-tuple of non-negative integers $\alpha=\left(\alpha_{1}, \cdots, \alpha_{m}\right)$ let

$$
L^{\alpha}=L_{1}^{\alpha_{1}} \cdots L_{m}^{\alpha_{m}}
$$

Our main result is

Theorem 2.1. Let $M^{2 m+1}$ be a $C^{\omega}$ CR manifold of nondegenerate Levi form. Let $\left\{L_{1}, \cdots, L_{m}\right\}$ be $C^{\omega}$ independent sections of the $C R$ structure bundle $\mathcal{V}$. Let $N$ be a $C^{\omega}$ real hypersurface in $\mathbb{C}^{n+1}, n \geq m$, defined by $r(z, \bar{z})=0$, where $r(z, \bar{z})$ is normalized as in (0.1). Let $f$ : $M \rightarrow N$ be a CR mapping. Suppose that for some positive integer $k$ the vectors $\left\{L^{\alpha} f:|\alpha| \leq k\right\}$ together with $(0, \cdots, 0,1)$ span $\mathbb{C}^{n+1}$. Then $f$ satisfies a complete system of order $2 k+1$. Thus, $f$ is determined by $2 k$-jet at a point and $f$ is $C^{\omega}$ provided that $f \in C^{2 k+1}$.

If $m=n, f: M \rightarrow N$ is a $\mathrm{CR}$ equivalence, then $\left\{L_{1} f, \cdots, L_{n} f\right\}$ together with $(0, \cdots, 0,1)$ span $\mathbb{C}^{n+1}$, thus $k=1$ and $f$ satisfies a complete system of order 3 . In the case $n>m$, the fact that $f$ is dertermined by finite jet at a point is the local rigidity of CR mappings. If $M$ is a real hypersurface in $\mathbb{C}^{m+1}$, a CR mapping $f$ extends holomorphically to a neighborhood of $M$ if and only if $f$ is analytic, thus, if $f$ satisfies the hypothesis of Theorem 2.1 then $\mathrm{f}$ extends holomorphically . One can also apply the argument of the Lewy-Pinchuk reflection principle [Forst] to $(2.4)$ of $\S 2$, to get the holomorphic extension of $f$.

\section{$\S 1$. Complete systems}

Let $f$ be a smooth $\left(C^{\infty}\right)$ mapping of an open subset $X$ of $\mathbb{R}^{n}$ into an open subset $U$ of $\mathbb{R}^{m}$. In this section we use superscripts for each components of vectors, thus $x=\left(x^{1}, \cdots, x^{n}\right)$ and $u=\left(u^{1}, \cdots, u^{m}\right)$ are the standard coordinates of $\mathbb{R}^{n}$ and $\mathbb{R}^{m}$, respectively, and $f(x)=$ $\left(f^{1}(x), \cdots, f^{m}(x)\right)$. 
Let $U_{k}$ be the space of all the different $k$-th order partial derivatives of the component of $f$ at a point $x$. Set $U^{(q)}=U \times U_{1} \times \cdots \times U_{q}$ be the Cartesian product space whose coordinates represent all the derivatives of a mapping $u=f(x)$ of all orders from 0 to $q$. A point in $U^{(q)}$ will be denoted by $u^{(q)}$.

The space $J^{q}(X, U)=X \times U^{(q)}$ is called the $q$-th order jet space of the space $X \times U$. If $f: X \rightarrow U$ is smooth, let $\left(j^{q} f\right)(x)=\left(x, f(x), \partial^{\alpha}(x)\right.$ : $|\alpha| \leq q)$, then $j^{q} f$ is a smooth section of $J^{q}(X, U)$ called the $q$-graph of $f$.

Consider a system of partial differential equations of order $q(q \geq 1)$ for unknown functions $u=\left(u^{1}, \cdots, u^{m}\right)$ of independent variables $x=$ $\left(x^{1}, \cdots, x^{n}\right)$,

$$
\Delta_{\lambda}\left(x, u^{(q)}\right)=0, \quad \lambda=1, \cdots, l,
$$

where $\Delta_{\lambda}\left(x, u^{(q)}\right)$ are smooth functions in their arguments. Then $\Delta=$ $\left(\Delta_{1}, \cdots, \Delta_{l}\right)$ is a smooth map from $X \times U^{(q)}$ into $\mathbb{R}^{l}$, so that the given system of partial differential equations describes the subset $\mathcal{S}_{\Delta}$ of zeros of $\Delta_{\lambda}$ in $X \times U^{(q)}$, called the solution subvariety of (1.1). Thus, a smooth solution of (1.1) is a smooth map $f: X \rightarrow U$ whose $q$-graph is contained in $\mathcal{S}_{\Delta}$.

A differential function $P\left(x, u^{(q)}\right)$ of order $q$ defined on $X \times U^{(q)}$ is a smooth function of $x, u$, and derivatives of $u$ up to order $q$. The total derivatives of $P\left(x, u^{(q)}\right)$ with respect to $x^{i}$ is the unique smooth function defined by

$$
D_{i} P\left(x, u^{(q+1)}\right):=\frac{\partial P}{\partial x^{i}}+\sum_{a=1}^{m} \sum_{J} u_{J, i}^{a} \frac{\partial P}{\partial u_{J}^{a}},
$$

where $J=\left(j_{1}, \cdots, j_{n}\right)$ is a multi-index such that $|J| \leq q$ and $J, i=\left(j_{1}, \cdots, j_{i}+1, \cdots, j_{n}\right)$. For each nonnegative integer $r$, the $r$ thprolongation $\Delta^{(r)}$ of the system $(1.1)$ is the system consisting of all the total derivatives of $(1.1)$ of order up to $r$. Let $\left(\Delta^{(r)}\right)$ be the ideal generated by $\Delta^{(r)}$ of the ring of differential functions on $X \times U^{(q+r)}$. If $\widetilde{\Delta} \in\left(\Delta^{(r)}\right)$ for some $r$, the equation

$$
\widetilde{\Delta}\left(x, u^{(q+r)}\right)=0
$$

is called a prolongation of(1.1). Note that any smooth solution of (1.1) must satisfy (1.2). If $k$ is the order of the highest derivative involved in $\widetilde{\Delta}$, we call (1.2) a prolongation of order $k$.

We now define the complete system. 
Definition 1.1. We say that a $C^{k}(k \geq q)$ solution $f$ of (1.1) satisfies a complete system of order $k$ if there exist prologations of (1.1) of order $k$

$$
\widetilde{\Delta}_{\nu}\left(x, u^{(k)}\right)=0, \quad \nu=1, \cdots, N
$$

which can be solved for all the $k$-th order partial derivatives as smooth functions of lower order derivatives of $f$, namely, for each $a=1, \cdots, m$ and for each multi-indix $J$ with $|J|=k$,

$$
f_{J}^{a}=H_{J}^{a}\left(x, f^{(p)}: p<k\right)
$$

for some function $H_{J}^{a}$ which is smooth in its arguments.

The idea of complete system is found in the so-called equivalence problem of E. Cartan: Let $G$ be a Lie-subgroup of $G L(n ; \mathbb{R})$ and $\pi: Y \rightarrow$ $E$ be a principal fibre bundle with the structure group $G$ over a manifold $E$ of dimension $n$. The equivalence problem is finding canonically a system of differential 1-forms

$$
\omega^{1}, \cdots, \omega^{N}, \quad \text { where } \quad N=n+\operatorname{dim} G,
$$

so that a mapping $f: E \rightarrow \tilde{E}$ preserves the $G$-structure if and only if there exists a mapping $F ; Y \rightarrow \tilde{Y}$, which is a lift of $f$, that is, $\tilde{\pi} \circ F=f \circ \pi$, and such that

$$
F^{*} \tilde{\omega}^{i}=\omega^{i}, \quad i=1, \cdots N,
$$

where $\tilde{\pi}: \tilde{Y} \rightarrow \tilde{E}$ is principal fibre bundle of the same structure group $G$ and $\tilde{\omega}^{i}$ are the corresponding 1-forms on $\tilde{Y}$. (see [Burns,],[BS]). (1.5) is called a complete system of invariants of the $G$-structure and (1.6) is a complete system of order 1 for $F$ in the sense of Definition 1.1. It turns out that (1.6) is equivalent to a complete system of order 2 for $f$ (see [H3]).

Now we recall that solving the given system of partial differential equations (1.1) is equivalent to finding an integral manifold of the corresponding exterior differential system

$$
d u_{I}^{a}-\sum_{i=1}^{n} u_{I, i}^{a} d x^{i}=0
$$

for all multi-index $I$ with $|I|<q$ and $a=1, \cdots, m$, with an independence condition $d x_{1} \wedge \cdots \wedge d x_{n} \neq 0$ on $\mathcal{S}_{\Delta}$ (see [BCGGG]). If a solution of (1.1) 
satisfies a complete system of order $k$ then we have the following Pfaffian system on $J^{k-1}(X, U)$ :

$$
\begin{cases}d u^{a}-\sum_{j=1}^{n} u_{j}^{a} d x^{j}=0, & \\ \vdots & |I|=k-2, \\ d u_{I}^{a}-\sum_{j=1}^{n} u_{I, j}^{a} d x^{j}=0, & |I|=k-1 . \\ d u_{I}^{a}-\sum_{i=1}^{n} H_{I, i}^{a} d x^{i}=0, & \end{cases}
$$

with an independence condition $d x^{1} \wedge \cdots \wedge d x^{n} \neq 0$, where $H_{I, i}^{a}$ are as in (1.4). Thus , a solution $u=f(x)$ of (1.1) of class $C^{k}$ satisfies a complete system of order $k$ if and only if

$$
(x) \mapsto\left(x, f(x), \partial_{J} f(x):|J| \leq k-1\right)
$$

is an integral manifold of the Pfaffian system (1.7). In particular, we have

Proposition 1.2. Let $f$ be a solution of (1.1) of class $C^{k}$. Suppose that $f$ satisfies a complete system (1.4), then $f$ is determined by $(k-1)$ jet at a point and $f$ is $C^{\infty}$. Furthermore, if (1.1) is real analytic and each $H_{J}^{a}$ is real analytic then $f$ is real analytic.

\section{$\S$ 2. Prolongation of CR mappings}

In this section we shall prove the following

Theorem 2.1. Let $M^{2 m+1}$ be a $C^{\omega}$ CR manifold of nondegenerate Levi form. Let $\left\{L_{1}, \cdots, L_{m}\right\}$ be $C^{\omega}$ independent sections of the $C R$ structure bundle $\mathcal{V}$. Let $N$ be a $C^{\omega}$ real hypersurface in $\mathbb{C}^{n+1}, n \geq m$, defined by $r(z, \bar{z})=0$, where $r(z, \bar{z})$ is normalized as in (0.1). Let $f$ : $M \rightarrow N$ be a CR mapping. Suppose that for some positive integer $k$ the vectors $\left\{L^{\alpha} f:|\alpha| \leq k\right\}$ together with $(0, \cdots, 0,1)$ span $\mathbb{C}^{n+1}$. Then $f$ satisfies a complete system of order $2 k+1$. Thus, $f$ is determined by $2 k$-jet at a point and $f$ is $C^{\omega}$ provided that $f \in C^{2 k+1}$.

Proof. $f=\left(f_{1}, \cdots, f_{n+1}\right)$ is a CR mapping of $M$ into $N$ if and only if 
$\bar{L}_{i} f_{j}=0, \quad$ for each $\quad i=1, \cdots, m$ and $j=1, \cdots, n+1$

(tangential Cauchy-Riemann equations)

and $r \circ f=0$, that is,

$$
f_{n+1}+\bar{f}_{n+1}+\sum_{j=1}^{n} \lambda_{j} f_{j} \bar{f}_{j}+\sum_{A, B} c_{A B} f^{A} \bar{f}^{B}=0
$$

where $f^{A}:=f_{1}^{a_{1}} \cdots f_{n+1}^{a_{n+1}}$ and the terms in the last summand are of weight $\geq 3$. Let $\alpha=\left(\alpha^{1}, \cdots, \alpha^{m}\right)$ be a $m$-tuple of non-negative integers. Apply $\overline{\bar{L}}^{\alpha}$ to (2.2), then by (2.1) we have

$$
\bar{L}^{\alpha} \bar{f}_{n+1}+\sum_{j=1}^{n} \lambda_{j} f_{j} \bar{L}^{\alpha} \bar{f}_{j}+\sum c_{A B} f^{A}\left(\bar{L}^{\alpha} \bar{f}^{B}\right)=0 .
$$

Since the set of vectors $\left\{\bar{L}^{\alpha} \bar{f}:|\alpha| \leq k\right\}$ and $(0, \cdots, 0,1)$ contains $(n+1)$ linearly independent vectors, we can solve (2.2) and (2.3) for $\left(f_{1}, \cdots, f_{n+1}\right)$ in terms of $\bar{L}^{\alpha} \bar{f},|\alpha| \leq k$, to get

$$
f_{j}=H_{j}\left(\bar{L}^{\alpha} \bar{f}:|\alpha| \leq k\right), \quad \text { for each } \quad j=1, \cdots, n+1,
$$

where each $H_{j}$ is an analytic function of the arguments in the parenthesis. we have

Let $\beta=\left(\beta_{1}, \cdots, \beta_{m}\right)$ be any multi-index. Apply $L^{\beta}$ to (2.4). Then

$$
L^{\beta} f_{j}=L^{\beta} H_{j}\left(\bar{L}^{\alpha} \bar{f}:|\alpha| \leq k\right) .
$$

Now let $T$ be a $C^{\omega}$ real vector field on $M$ which is transversal to the $\mathcal{V} \oplus \overline{\mathcal{V}}$, so that the set $\left\{T, L_{j}, \bar{L}_{j}, j=1, \cdots, m\right\}$ forms a basis of the complexified tangent space of $M$. Let $\left[L_{j}, \bar{L}_{k}\right]=\sqrt{-1} \rho_{j \bar{k}} T \bmod (\mathcal{V}, \overline{\mathcal{V}})$. Then $\left(\rho_{j \bar{k}}\right), \quad j, k=1, \cdots, m$ is a non-degenerate hermitian matrix. We may assume that $\left[\rho_{j \bar{k}}(0)\right]$ is diagonal at the refence point $0 \in M$. In the right hand side of (2.5), each time we apply $L_{i}$ to $H_{j}\left(\bar{L}^{\alpha} \bar{f}:|\alpha| \leq k\right)$, computations by chain rule show that $T$-directional derivatives occurs when commuting $L$ and $\bar{L}$, and by (2.1) the total order of the derivatives 
remains $\leq k$, for example,

$$
\begin{aligned}
\bar{L}_{1} L_{1} f_{j} & =\left(L_{1} \bar{L}_{1}-\left[L_{1}, \bar{L}_{1}\right]\right) f_{j} \\
& =\left\{L_{1} \bar{L}_{1}-\left(\sqrt{-1} \rho_{1} \overline{1} T+\sum_{i=1}^{m}\left(a_{i} L_{i}+b_{i} \bar{L}_{i}\right)\right)\right\} f_{j}
\end{aligned}
$$

for some functions $a_{i}$ and $b_{i}$

$$
=-\sqrt{-1} \rho_{1 \overline{1}} T f_{j}+\sum_{i=1}^{m} a_{i} L_{i} f_{j} \quad \text { by }(2.1) \text {. }
$$

Now we introduce notations : for each pair of non-negative integers $(p, q)$ with $p \geq q$, let $C_{p}$ be the set of $C^{\omega}$ functions in the arguments

$$
T^{t} L^{\alpha} f_{j}: t+|\alpha| \leq p, \quad j=1, \cdots, n+1
$$

and $C_{p, q}$ be the subset of $C_{p}$ of $C^{\omega}$ functions in the arguments

$$
T^{t} L^{\alpha} f_{j}: t+|\alpha| \leq p, \quad t \leq q, \quad j=1, \cdots, n+1,
$$

and let $\bar{C}_{p}, \bar{C}_{p, q}$ be the complex conjugate of $C_{p}$ and $C_{p, q}$, respectively. Then (2.5) implies that $L^{\beta} f_{j} \in \bar{C}_{k}, \quad$ for any multi-index $\beta=\left(\beta_{1}, \cdots\right.$, $\left.\beta_{m}\right)$.

In particular, for each $i=1, \cdots, m$

$$
L_{i} f_{j} \in \bar{C}_{k} \text {. }
$$

Apply $\bar{L}_{i}$ to (2.7), then by the same calculation as in (2.6) we have

$$
T f_{j} \in \bar{C}_{k+1, k}
$$

Similarly, for each $i, k=1, \cdots, m$, we have

$$
L_{k} L_{i} f_{j} \in \bar{C}_{k}
$$

Apply $\bar{L}_{k}$ to $(2.9)$, then by $(2.7),(2.8)$ and (2.9) we have

$$
T L_{i} f_{j} \in \bar{C}_{k+1, k} \text {. }
$$

Then by induction on $|\alpha|$, we have

$$
T L^{\alpha} f_{j} \in \bar{C}_{k+1, k}
$$

Now apply $\bar{L}_{i} \bar{L}_{k}$ to $(2.9)$, then by $(2.7)-(2.11)$ we have

$$
T^{2} f_{j} \in \bar{C}_{k+2, k}
$$


and by induction on $|\alpha|$, we have

$$
T^{2} L^{\alpha} f_{j} \in \bar{C}_{k+2, k}
$$

Then by induction on $t$, we have

$$
T^{t} L^{\alpha} f_{j} \in \bar{C}_{k+t, k}, \quad \text { for each } \quad j=1, \cdots, n+1,
$$

which shows that

$$
C_{p, q} \subset \bar{C}_{k+q, k}, \quad \text { for any pair }(p, q) \text { with } p \geq q \text {. }
$$

Taking the complex conjugate of (2.15), we have

$$
\bar{C}_{p, q} \subset C_{k+q, k}, \quad \text { for any pair }(p, q) \text { with } p \geq q .
$$

In paticular, if $q=k$

$$
\bar{C}_{p, k} \subset C_{2 k, k} \text { for all } p \geq k \text {. }
$$

Substitute (2.17) in (2.15), to get

$$
C_{p, q} \subset C_{2 k, k}, \quad \text { for any pair }(p, q) \text { with } p \geq q \text {. }
$$

In particular, we have

$$
C_{2 k+1} \subset C_{2 k}
$$

Now consider the derivatives $T^{t} L^{\alpha} \bar{L}^{\beta} f_{j}$, where $t+|\alpha|+|\beta|=2 k+1$. If $|\beta| \neq 0$, this is zero by (2.1). If $|\beta|=0$, then (2.19) shows that $T^{t} L^{\alpha} f_{j}, t+|\alpha|=2 k+1$, can be expressed as a $C^{\omega}$ function in the arguments $T^{t} L^{\beta} f_{j}: t+|\beta| \leq 2 k$, thus, $f$ satisfies a complete system of order $2 k+1$, which completes the proof. 


\section{References}

[BCGGG] R. Bryant, S. S. Chern, R. B. Gardner, H. Goldschmidt, and P. Griffiths, "Exterior differential systems", Springer-Verlag, New-York, 1986.

[Burns] D. Burns, CR Geometry, U. of Michigan Lecturenote (1980).

[BS] D. Burns and S. Shnider, Real hypersurfaces in complex manifolds, Proc. Symp. Pure math., 30 (1976), 141-167.

[CHY] C. K. Cho, C. K. Han and J. N. Yoo, Complete differential systems for certain isometric immersions of Riemannian manifolds, Comm. Korean Math. Soc., 8 (1992), 315-328.

[Ch-M] S. S. Chern and J. Moser, Real hypersurfaces in complex manifolds, Acta Math., 133 (1974), 219-271.

[Forst] F. Forstnerič, Proper holomorphic mappings : a survey, in "Several Complex Variables : Proc. of the Mittag-Leffler Institute, 1987-1988 (ed. John Erik Fornaess)", Princeton Univ. Press, Princeton, 1993, pp. 297-363.

[H1] C. K. Han, Analyticity of CR equivalence between real hypersurfaces in $\mathbb{C}^{n}$ with degenerate Levi form, Invent. Math., 73 (1983), 51-69.

[H2] Regularity and uniqueness of certain systems of functions annihilated by a formally integrable system of vector fields, Rocky Mt. J. of Math., 18, No. 4 (1988), 767-783.

[H3] Analyticity and rigidity of CR immersions, To appear.

Chong-Kyu Han

Department of Mathematics,

Seoul National University,

Seoul, 151-742,

Korea.

ckhan@math.snu.ac.kr

Jae-Nyun Yoo

Department of Mathematics

Pohang University of Science and Technology

Pohang, 790-600,

Korea.

jnyoo@posmath.postech.ac.kr 